\title{
Age at diagnosis is a determinant factor of renal cell carcinoma- specific survival in patients treated with nephrectomy
}

\author{
Pierre. I. Karakiewicz, MD; ${ }^{*}$ Claudio Jeldres, MD; ${ }^{*}$ Nazareno Suardi, MD; ${ }^{*}$ George C. Hutterer, MD; ${ }^{*}$ \\ Paul Perrotte, MD; $;^{+}$Umberto Capitanio, MD; ${ }^{*}$ Vincenzo Ficarra, MD; ${ }^{\neq}$Luca Cindolo, MD; ${ }^{\xi}$ \\ Alexandre de La Taille, MD;" Jacques Tostain, MD; ${ }^{* *}$ Peter F. Mulders, MD; ${ }^{+\dagger}$ Laurent Salomon, MD;" \\ Richard Zigeuner, MD,; Luigi Schips, MD, ${ }^{\prime \neq}$ Denis Chautard, MD, ${ }^{\xi \mathcal{S}}$ Antoine Valeri, MD;/7/ \\ Eric Lechevallier, MD;/7/ Jean-Luc Descots, MD;"7/7 Herve Lang, MD;" Arnaud Mejean, MD;" \\ Gregory Verhoest, MD; ${ }^{* *}$ Jean-Jacques Patard, MD I/7**** $^{*{ }^{*}}$
}

See related article on page 618

\section{ABSTRACT}

Objective: Based on combined data for 4880 patients, 2 previous studies reported that advanced age is a predictor of increased renal cell carcinoma-specific mortality (RCC-SM). We explored the effect of age in cubic spline analyses to identify the age groups with the most elevated risk for renal cell carcinoma (RCC).

Methods: Our study included 3595 patients from 14 European centres who had partial or radical nephrectomies. We used the Kaplan-Meier method to compile life tables, and we performed Cox regression analyses to assess RCC-SM. Covariates included age at diagnosis, sex, TNM (tumour, node, metastasis) stage, tumour size, Fuhrman grade, symptom classification and histological subtype.

Results: Age ranged from 10 to 89 (mean 63, median 67) years. The median duration of follow-up was 2.9 years. The median survival for the cohort was 13.4 years. Stage distribution was as follows: 1915 patients (53.3\%) had stage I disease, 388 (10.8\%) had stage II, 895 (24.9\%) had stage III and 397 (11.0\%) had stage IV disease. In multivariate analyses, we coded age at diagnosis as a cubic spline, and it achieved independent predictor status $(p<0.001)$. The risk of RCC-SM was lowest among patients younger than 50 years. We observed an increase in RCC-SM until the age of 50, at which point the level of risk reached a plateau. We observed a second increase among patients aged 75-89 years. We found similar patterns when we stratified patients according to the 2002 American Joint Committee on Cancer (AJCC) stages.

Conclusion: The effect of age shows prognostic significance and indicates that follow-up and possibly secondary treatments might need to be adjusted according to the age of the patient.

Can Urol Assoc J 2008;2(6):610-7

\section{Introduction}

Renal cell carcinoma (RCC) accounts for $3 \%$ of cancers in adults and $85 \%$ of all primary renal cancers. ${ }^{1}$ The 5 -year survival rate for all stages of RCC has improved in recent years owing to an important stage migration, whereby most patients receive diagnoses of localized disease. ${ }^{2}$ Nonetheless, advanced disease, either distant or nodal metastases, is diagnosed in nearly $25 \%$ of contemporary patients. ${ }^{2}$ Recently, 2 groups of investigators assessed the effect of age on renal cell carcinoma-specific mortality (RCC-SM) in a combined cohort of 4880 patients. ${ }^{3-5}$ Although these findings are interesting, the cut-offs used to assess the effect of age were not based on specific RCC biology. Instead they were adapted from literature addressing other types of cancer. ${ }^{6,7}$

We sought to re-examine the effect of age on RCC-SM in a large multi-institutional cohort of 3595 patients using cubic spline analyses, which help identify data-driven relations, instead of using predefined cut-offs or forcing linear relations.

\section{Methods}

\section{Patient population}

Between 1980 and 2000, a total of 3595 patients at 14 European centres underwent either a partial or radical nephrectomy for RCC. For each patient, records provided information on age at diagnosis, sex, TNM (tumour, node metastasis) stage, nodal status, tumour size, Fuhrman grade, symptom classification and histological subtype. Moreover, each record included dates of follow-up and specific survival data. 
Age a factor in survival in patients treated with nephrectomy

\section{Clinical and pathological evaluation}

We classified tumours according to the 2002 TNM staging system and according to Fuhrman grade. Tumour size, defined as the greatest diameter in centimetres, was based on pathological specimens. Prior to study inclusion, we stratified histological subtypes according to the 2002 American Joint Committee on Cancer (AJCC) and Union Internationale Contre le Cancer (UICC) classifications; we included only patients with tumours of clear, chromophobe or papillary cell histology. ${ }^{8}$ Symptoms were recorded at each of the participating institutions according to a previously validated coding scheme. ${ }^{9}$ Diagnoses in asymptomatic patients were typically based on routine imaging. Patients with local symptoms reported lumbar pain, hematuria or a palpable mass. Patients with systemic symptoms reported fatigue, weight loss, fever, night sweats or cough. We staged patients preoperatively based on computed tomography (CT) scans of the abdomen, pelvis and chest; chest radiographs; serum electrolytes; and liver function test results. We detected the presence of nodal metastases based on findings from lymphadenectomy. All patients underwent hilar lymphadenectomies that included all lymph nodes on the ipsilateral side of the great vessels. Select patients, based on surgeon preference, underwent more extensive lymphadenectomies that included interaortocaval lymph nodes. Since the number of nodes removed, the number of metastatic nodes and other specific details about the extent, technique and pathological handling of the lymphadenectomy specimens were not prospectively collected at all centres, we could not include these details in our analyses. Nonetheless, in all patients, the presence of nodal metastases was confirmed pathologically; the presence of distant metastases was confirmed radiographically.

Follow-up consisted of 1 postoperative baseline visit and subsequent visits taking place at least every 6 months for a minimum of 2 years, after which time the minimum follow-up consisted of annual visits. At each visit, CT scans of the abdomen and either CT scans of the chest or chest radiographs were obtained.

We extracted the cause of death from the medical charts or the death certificates. Renal cell carcinoma-specific mortality included deaths that were directly attributable to renal cancer.

\section{Statistical analyses}

Our statistical analyses relied on life tables compiled using the Kaplan-Meier method and Cox regression analyses, which targeted the time from radical nephrectomy to death. In multivariate Cox regression models, the covariates included age at diagnosis, sex, TNM stage, nodal status, tumour size, Fuhrman grade, symptom classification and histological subtype. Our analyses of the effect of age at diagnosis on RCC-SM relied on cubic splines to allow for nonlinear effects. The multivariate effect of age at diagnosis on RCC-SM was represented graphically, because the coefficients of the cubic splines cannot be directly interpreted. The nonlinear quality of the cubic spline analyses allows the curve that depicts age to assume a data-driven shape. This is not predefined by specific age strata or other preconceived considerations that may inflate or hide the effect of age on RCC-SM. This quality of cubic spline analysis is a major advantage of this technique over standard linear or stratified analyses. We performed all tests on the entire cohort, and we then repeated the tests in subgroups defined according to AJCC stages. We performed all statistical tests using S-PLUS Professional, version 1 (MathSoft Inc.). All tests were 2-sided with a significance level set at $p=0.05$.

\section{Results}

Table 1 summarizes the descriptive characteristics of the study population. The mean age at diagnosis was 63 (median 67) years. Most patients were male $(66.7 \%)$. The TNM stage distribution was as follows: 1915 patients (53.3\%) had stage I disease, $388(10.8 \%)$ had stage II, $895(24.9 \%)$ had stage III and $397(11.0 \%)$ had stage IV disease. In the entire cohort, 261 patients $(7.3 \%)$ had nodal metastases and 355 (9.9\%) had distant metastases. The mean tumour size was 6.2 (median 5.2 ) cm. The most frequent histological subtype was clear cell $(88.1 \%)$. Most of the patients were asymptomatic at the time of diagnosis (61.0\%). Overall, the median duration of follow-up was 2.9 (range $0.1-24.9)$ years and the median duration of followup for censored patients was 3.0 years. Finally, 693 patients (19.3\%) died of RCC during the follow-up period.

Figure 1 shows the Kaplan-Meier plot of 
RCC-specific survival after radical nephrectomy. 15 years after the procedure. The median survival In life table analyses, the probability estimates of was 13.4 years.

survival after radical nephrectomy were $79.6 \%$

Table 2 shows univariate and multivariate Cox at 5 years, $62.6 \%$ at 10 years and $46.4 \%$ at regression analyses addressing the association

\section{Table 1. Variables for 3595 patients treated for renal cell carcinoma}

\begin{tabular}{|c|c|c|c|c|c|c|c|c|c|c|}
\hline \multirow{3}{*}{$\begin{array}{l}\text { Variable } \\
\text { Total }\end{array}$} & \multirow{2}{*}{\multicolumn{2}{|c|}{$\begin{array}{c}\text { Overall } \\
\text { no. }(\%)^{*}\end{array}$}} & \multicolumn{8}{|c|}{ Stage, no. $(\%)^{*}$} \\
\hline & & & \multicolumn{2}{|c|}{ I } & \multicolumn{2}{|c|}{ ॥ } & \multicolumn{2}{|c|}{ III } & \multicolumn{2}{|c|}{ IV } \\
\hline & 3595 & $(100.0)$ & 1915 & (53.3) & 388 & (10.8) & 895 & (24.9) & 397 & (11.0) \\
\hline \multicolumn{11}{|l|}{ Age } \\
\hline Mean (median) & 62.8 & (67.0) & 62.9 & $(68.0)$ & 61.9 & (59.0) & 63.1 & $(63.0)$ & 62.7 & (61.0) \\
\hline Range & \multicolumn{2}{|c|}{$10-89$} & \multicolumn{2}{|c|}{$10-87$} & \multicolumn{2}{|c|}{$27-87$} & \multicolumn{2}{|c|}{$15-88$} & \multicolumn{2}{|c|}{ 28-89 } \\
\hline \multicolumn{11}{|l|}{ Sex } \\
\hline Male & 2397 & (66.7) & 1270 & (66.3) & 247 & (63.7) & 598 & (66.8) & 282 & (71.0) \\
\hline Female & 1198 & (33.3) & 645 & (33.7) & 141 & (36.3) & 297 & (33.2) & 115 & $(29.0)$ \\
\hline \multicolumn{11}{|l|}{ Clinical stage } \\
\hline T1 & 1976 & $(55.0)$ & 1915 & $(100.0)$ & & - & 19 & $(2.1)$ & 42 & (10.6) \\
\hline T1a & 253 & (12.8) & 1129 & $(59.0)$ & & - & 5 & $(0.5)$ & 11 & (2.3) \\
\hline $\mathrm{T} 1 \mathrm{~b}$ & 1723 & (87.2) & 786 & (41.9) & & 14 & $(1.6)$ & 31 & (8.3) \\
\hline T2 & 442 & (12.3) & \multicolumn{2}{|c|}{-} & 338 & (100.0) & 18 & $(2.0)$ & 37 & (9.3) \\
\hline T3 & 1116 & (31.0) & \multicolumn{2}{|c|}{-} & \multicolumn{2}{|c|}{-} & 858 & (95.9) & 258 & $(65.0)$ \\
\hline T4 & 60 & (1.7) & \multicolumn{2}{|c|}{ - } & \multicolumn{2}{|c|}{ - } & \multicolumn{2}{|c|}{-} & 60 & $(15.1)$ \\
\hline \multicolumn{11}{|l|}{ Nodal metastases } \\
\hline No & 3334 & (92.7) & & & & - & 768 & (85.8) & 263 & $(66.2)$ \\
\hline N1 & 234 & (6.5) & & & & - & 127 & $(14.2)$ & 107 & $(27.0)$ \\
\hline N2 & 27 & (0.8) & & & & - & 0 & $(0.0)$ & 27 & (6.8) \\
\hline Metastases & & & & & & & & & & \\
\hline Mo & 3240 & (90.1) & & & & - & & - & 42 & (10.6) \\
\hline M1 & 355 & (9.9) & & & & - & & - & 355 & (89.4) \\
\hline Tumour size, cm & & & & & & & & & & \\
\hline Mean (median) & 6.2 & $(5.2)$ & 4.0 & (4.0) & 9.8 & $(9.0)$ & 7.8 & (7.5) & 9.3 & (9.0) \\
\hline Range & 0.5 & -25 & & & & -20 & 1.0 & -20 & 2.0 & -25 \\
\hline Fuhrman grade & & & & & & & & & & \\
\hline । & 886 & $(24.6)$ & 771 & (40.3) & 40 & (10.3) & 57 & (6.4) & 18 & (4.5) \\
\hline$\|$ & 1470 & $(40.9)$ & 860 & (44.9) & 230 & (59.3) & 300 & (33.5) & 80 & $(20.2)$ \\
\hline III & 1017 & (28.3) & 268 & $(14.0)$ & 103 & $(26.5)$ & 448 & $(50.1)$ & 198 & (49.9) \\
\hline IV & 222 & (6.2) & 16 & (0.8) & 15 & (3.9) & 90 & $(10.1)$ & 101 & $(25.4)$ \\
\hline Histological type & & & & & & & & & & \\
\hline Clear cell & 3168 & (88.1) & 1648 & $(86.1)$ & 324 & (83.5) & 822 & (91.8) & 374 & (94.2) \\
\hline Papillary & 326 & (9.1) & 211 & (11.0) & 41 & $(10.6)$ & 52 & (5.8) & 22 & (5.5) \\
\hline Chromophobe & 101 & (2.8) & 56 & (2.9) & 23 & $(5.9)$ & 21 & (2.3) & 1 & $(0.3)$ \\
\hline $\begin{array}{l}\text { Symptom } \\
\text { classification }\end{array}$ & & & & & & & & & & \\
\hline Asymptomatic & 2192 & $(61.0)$ & 1506 & (78.6) & 183 & $(47.2)$ & 408 & $(45.6)$ & 95 & (23.9) \\
\hline Local & 949 & $(26.4)$ & 357 & (18.6) & 163 & $(42.0)$ & 322 & $(36.0)$ & 107 & $(27.0)$ \\
\hline Systemic & 454 & $(12.6)$ & 52 & $(2.7)$ & 42 & (10.8) & 165 & (18.4) & 195 & $(49.1)$ \\
\hline Follow-up, yr & & & & & & & & & & \\
\hline Mean (median) & 4.0 & (2.9) & 3.8 & $(2.8)$ & 4.6 & $(4.0)$ & 4.2 & (3.0) & 4.2 & (2.8) \\
\hline Range & 0.10 & -24.9 & 0.10 & 21.6 & 0.01 & -18.7 & 0.10 & -24.9 & 0.10 & -20.1 \\
\hline
\end{tabular}


Age a factor in survival in patients treated with nephrectomy

between age at diagnosis and RCC-SM after radical nephrectomy for RCC. In univariate analyses, age at diagnosis $(p<0.001)$ and all the other variables $(p \leq 0.032)$ were statistically significant predictors of RCC-SM. Moreover, age at diagnosis achieved independent predictor status in the overall cohort $(p<0.001)$ and in all subgroup analyses that assessed the effect of age on RCC-SM according to AJCC stages $(p \leq 0.033)$. Pathological stage, nodal status, distant metastases status, Fuhrman grade and symptom classification also reached independent predictor status in the overall analysis $(p \leq 0.005)$. Conversely, some variables failed to reach independent predictor status in some of the subgroup analyses (Table 2).

The graphic representation of our multivariate analysis on the effect of age at diagnosis on RCC-SM is shown in Figure 2. The triphasic curve indicates that patients aged 50-75 years $(n=2176$, $60.5 \%$ ) were at intermediate risk for RCC-SM. Conversely, the risk for RCC-SM among patients aged 75 years or older $(n=697,19.4 \%$ ) was up to 7 times greater than among those aged 5075 years. Finally, patients younger than 50 years $(n=722,20.1 \%)$, were at the lowest risk for RCC-SM, which may be up to $90 \%$ lower than that of patients aged $50-75$ years.

Figure 3 shows the effect of age on RCC-SM according to AJCC stages. The graphic representation of the multivariate effect of age on RCC-SM demonstrated virtually similar patterns, where the youngest individuals were at the lowest risk for RCC-SM and the oldest individuals were at the highest risk (Fig. 3). The decrease in risk was most pronounced among young patients with stage II or III disease. Conversely, the increase in risk for RCC-SM was most pronounced in older patients with stage I, III or IV disease.

\section{Discussion}

The effect of age on RCC-SM was first examined by Lieber and colleagues, ${ }^{10}$ who reviewed the effects of different variables that affected the prognosis for young adults with RCC. The authors demonstrated that pathological stage and preoperative weight loss were significantly associated with survival. However, age did not reach prognostic significance. Meanwhile, other investigators have also examined the effect of age on various outcomes in other solid organ cancers., ${ }^{7,11}$ For example,
Gajdos and colleagues ${ }^{11}$ showed that, despite aggressive treatment, young patients with breast carcinoma had higher rates of local and distant failure than older patients. Moreover, Parramore and colleagues ${ }^{7}$ showed that younger patients with colon cancer often had less favourable prognoses than older patients. They stated that younger patients ( $\leq 40$ years) were more likely to present with advanced-stage disease than older patients. Therefore age is an established unfavourable prognostic marker in some solid tumours.

Despite the initial interest in age as a predictor of RCC-SM, only 3 groups of investigators revisited this topic since Lieber and colleagues reported their negative findings. $3,10,12,13$ Recently, 2 studies indicated that better survival could be expected in young patients and that age was an independent predictor of RCC-SM. ${ }^{3,5}$ These 2 studies involved 106 and 4774 patients, respectively. Both used an age cutoff of 40 years, which was consistent with cut-offs tested in previous studies of RCC and with cut-offs used in studies of other cancers. ${ }^{714}$ However, no biological basis supports the validity of this cut-off in RCC. Based on the sample size and methodological limitations of the previous studies, we sought to assess the effect of age in one of the largest multiinstitutional cohorts of patients treated with either partial or radical nephrectomies. We decided to explore this effect using an approach that was methodologically more advanced, namely, cubic spline analyses. Cubic splines enable the modelling of nonlinear effects. Unlike continuous or categorical coding, cubic splines do not assume any data

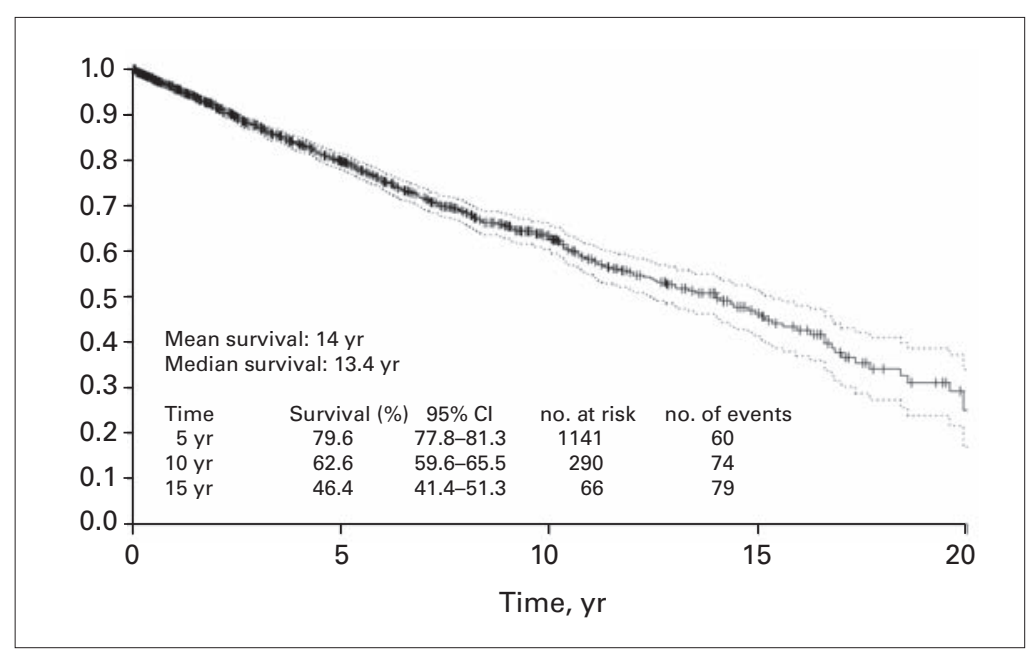

Fig. 1. Renal cell carcinoma-specific survival after nephrectomy. $\mathrm{Cl}$ = confidence interval. 
coding and do not force any predetermined relation such as linear effect. Instead, cubic splines are entirely data-driven. The cubic splines coefficients cannot be interpreted; however, the multivariate effect of variables coded as cubic splines can be displayed graphically so their effect after adjusting for all covariates can be visualized. We applied this methodology to our data set. This approach obviated the need to define cut-offs or create categories. Similarly, this approach also obviated the need to force a linear relation between age and RCC-SM.

Our analyses demonstrated that young patients are indeed at lower risk for RCC-SM. Despite this initial agreement with other studies, the multivariate analyses of the effect of age coded as a cubic spline demonstrated that the RCC-SM among young patients worsens up to the age of 50 years. Subsequently, the risk reaches a plateau, and another breakpoint can be observed at age 75 . At this point, the prognosis again steeply worsens. If the plateau is considered to be the reference point, young patients may be at an up to $87 \%$ lower risk for RCC-SM than those aged 50-75 years. Conversely, the breakpoint at age 75 indicates a rapidly increasing risk of RCC-SM beyond that age, which may reach an up to 7.4-fold increase compared with individuals aged $50-75$ years. These trends account for the multivariate effect of other covariates, which are held constant.

We stratified our cohort into 4 groups based

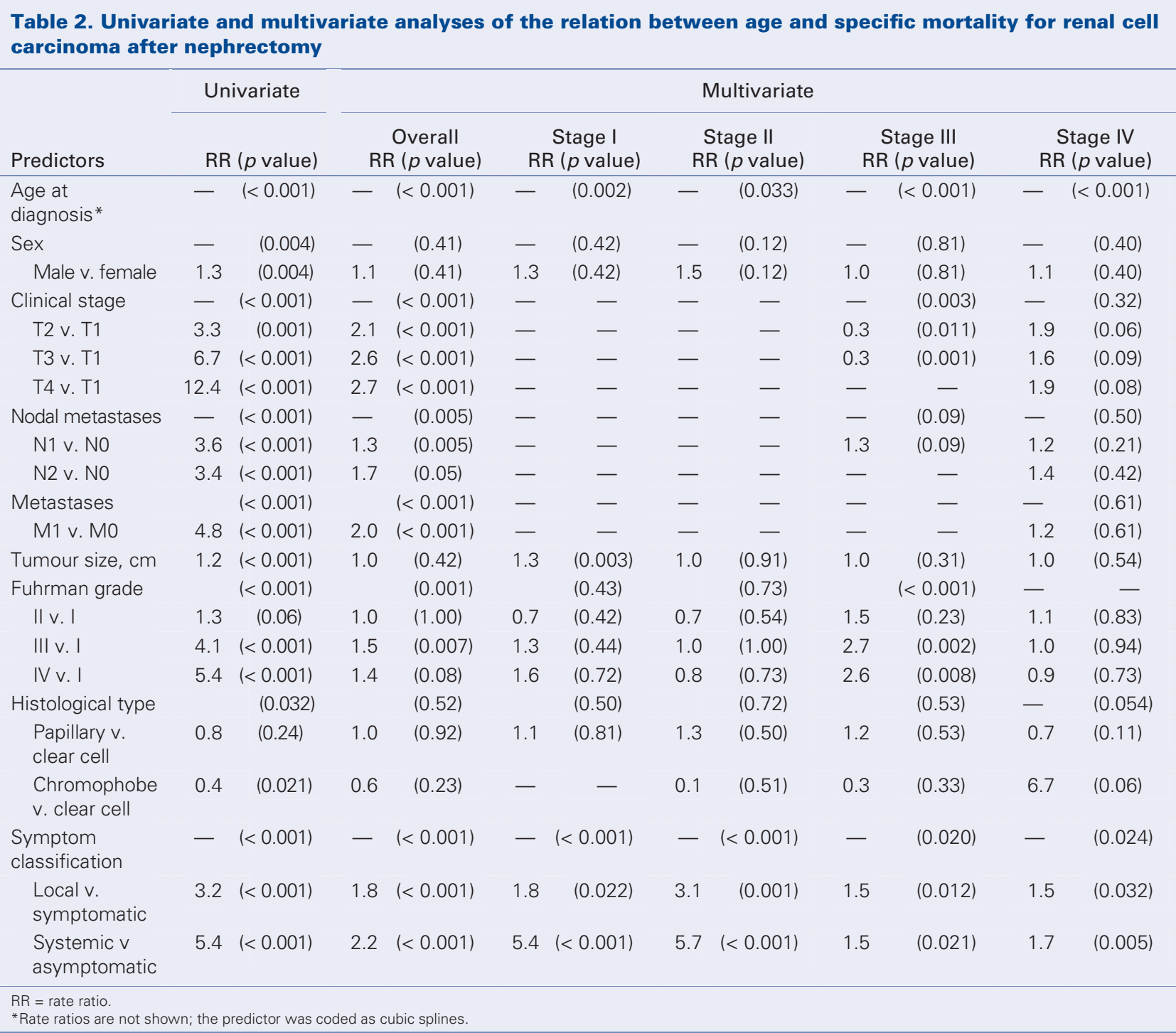


Age a factor in survival in patients treated with nephrectomy

on AJCC stages to investigate the multivariate effect of age according to these stages. Our analyses revealed the same trends: young patients had better prognosis, and the oldest patients had the worst prognosis. The plateau became more curvilinear in these analyses; however, the first breakpoint persisted between 50 and 55 years, and the second breakpoint remained between 70 and 75 years.

Unfortunately, we cannot directly compare the results of our study with those in other cohorts becaused none used similar methodology. Despite the methodological distinctness of our study, we did corroborate the findings of Sánchez-Ortiz and colleagues $^{3}$ and of Taccoen and colleagues, ${ }^{4}$ namely, that young age exerts a protective effect against RCC-SM. The novelty of our findings resides in the breakpoints that differ from previous studies that used the age of 40 years as a cut-off. Moreover, we identified a second breakpoint that shows a worsening of prognosis in older patients, despite holding all other covariates constant. This detrimental effect of advanced age was not previously known.

Our findings have 2 important implications. First, young patients should be reassured about their prognosis because, stage for stage and grade for grade, they may anticipate better control of their cancer. Conversely, older patients should be advised that their disease may progress more rapidly. Age-specific adjustments may be recommended for the delivery of adjuvant therapy, in follow-up frequency and possibly in the type of adjuvant therapy. The threshold and indications for adjuvant therapy are being investigated in 2 large randomized trials (EORTC protocol 30947 trial and ECOG-E2805 trial). These studies will hopefully provide valid answers to the question of which patients should receive adjuvant treatment. Until their completion, it might be postulated that the threshold for the delivery of adjuvant treatment should be lower in older patients. However, before our findings are implemented into clinical decisionmaking, further studies are needed to corroborate the observed effect of age on RCC-SM.

Worse prognosis in older patients also indicates that follow-up should be adjusted according to age; older patients should be followed-up more frequently and possibly undergo more detailed imaging studies.

Despite its strengths, our study has several weaknesses. First, the lack of standardization in the extent of lymphadenectomy represents a limitation. Owing to variability in lymphadenectomy, some patients may have been understaged if the extent of their surgery was suboptimal. Understaging may have contributed to spuriously worse survival outcomes. Second, the lack of central pathology review may have introduced interobserver biases. For example, tumour grades may have been interpreted differently by different pathologists. Third, we obtained no detail on the handling or evaluation of pathological specimens. Fourth, we did not have all the information about the adjuvant and/or salvage treatment regimens of the included patients. Some received adjuvant immunotherapy, whereas others received immunotherapy at the time of relapse. Some underwent experimental chemotherapy, whereas others received only the best supportive care. It is unlikely that adjuvant or salvage therapies have contributed to a significantly longer survival, because most historic regimens are associated with a dismal effect on survival. ${ }^{15-18}$ Despite these limitations, our findings are novel and offer a new perspective about the effect of age on RCC prognosis. In addition, the sample size makes our findings highly generalizable. Finally, the novel modelling techniques in the form of cubic splines offer a new alternative to standard data analyses. In conclusion, the effect of age shows prognostic significance and indicates that follow-up and possibly secondary treatments might need to be adjusted according to the age of the patient.

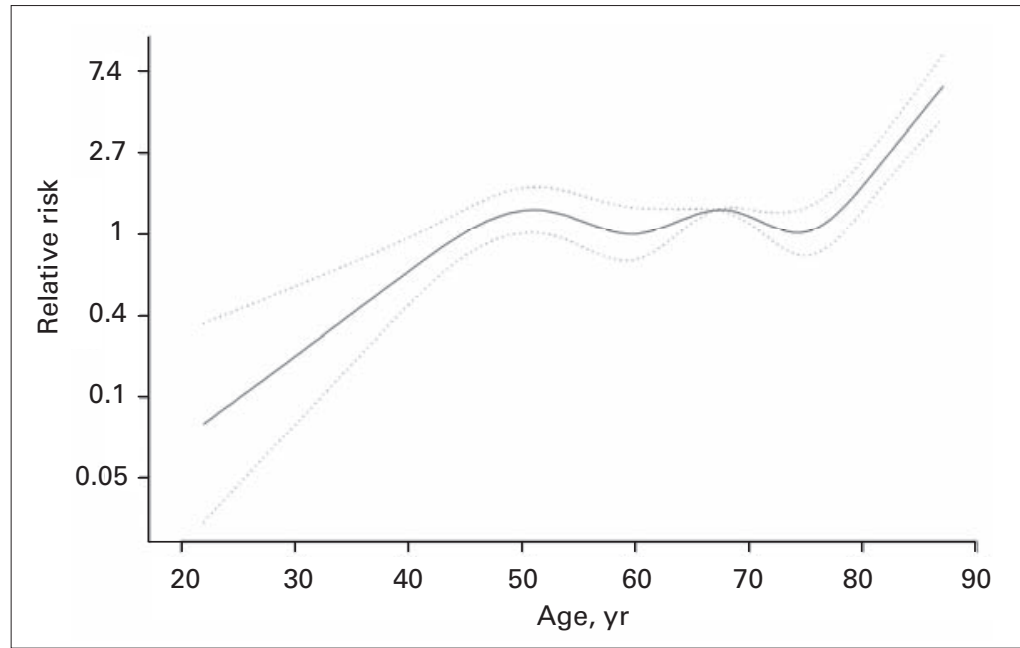

Fig. 2. Overall multivariate effect of age at diagnosis on renal cell carcinoma-specific survival after partial or radical nephrectomy according to American Joint Committee on Cancer stages. The solid lines represent the relation between age (coded as cubic splines) and the rate (relative risk) of renal cell carcinoma-specific mortality after nephrectomy. Dotted lines represent the $95 \%$ confidence interval. 
Karakiewicz et al.

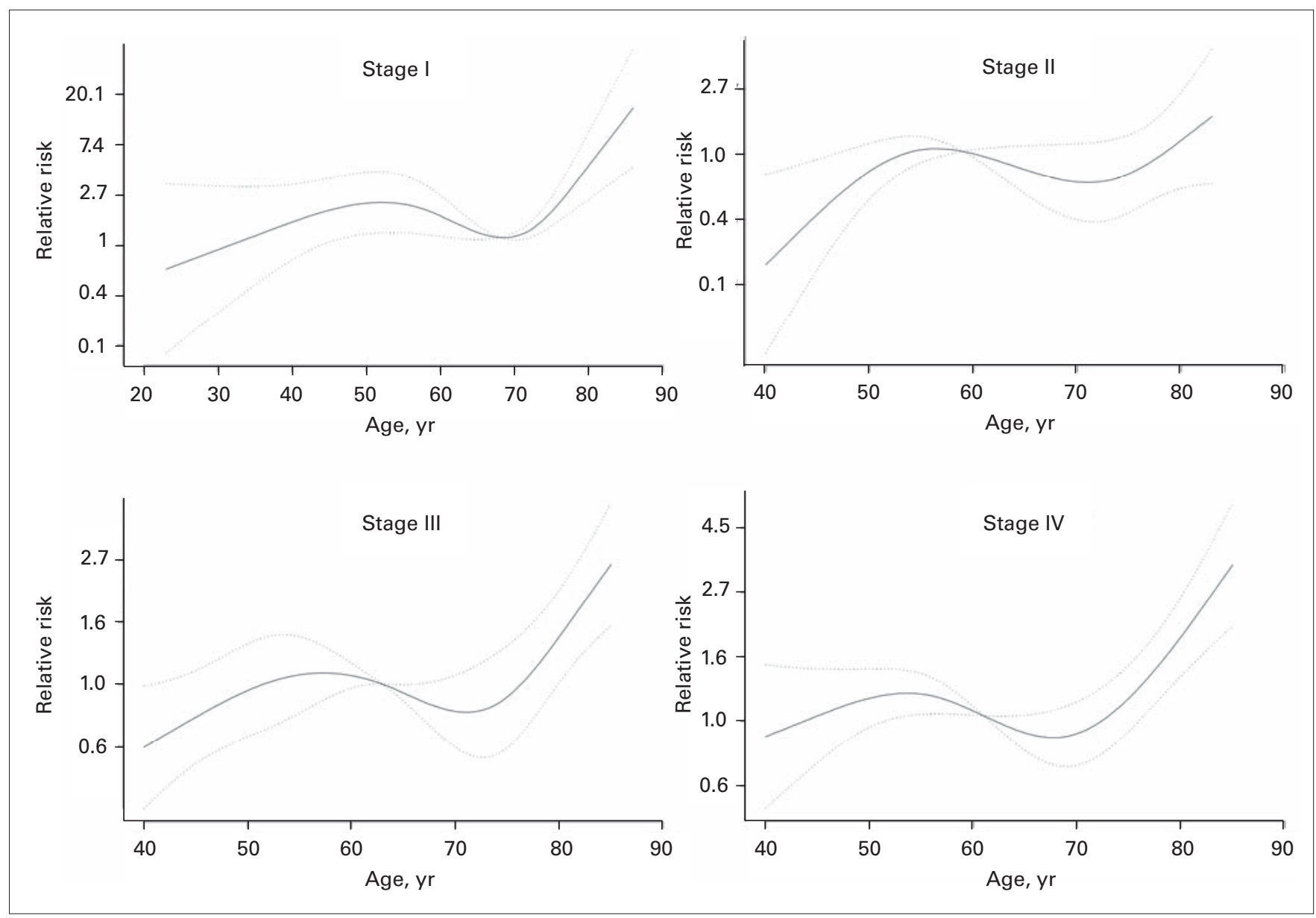

Fig. 3. Multivariate effect of age on renal cell carcinoma-specific survival after nephrectomy, according to American Joint Committee on Cancer stages. The solid lines represent the relation between age (coded as cubic splines) and the rate (relative risk) of renal cell carcinoma-specific mortality after nephrectomy. Dotted lines represent the $95 \%$ confidence interval.

From the *Cancer Prognostics and Health Outcomes Unit, University of Montréal Health Centre, the †Department of Urology, University of Montréal, Montréal, Que., the ‡Department of Urology, University of Padova, Padova, Italy, the §Department of Urology, S. Pio da Pietrelcina Hospital, Vasto, Italy, the IDepartment of Urology, Henri Mondor University Hospital, Creteil, France, the ${ }^{* *}$ Department of Urology, St. Etienne University Hospital, St. Etienne, France, the ${ }^{\dagger \dagger}$ Department of Urology, Radboud University Nijmegen Medical Center, Nijmegen, the Netherlands, the 㧊Department of Urology, Graz Medical University, Graz, Austria, the $\S^{\S}$ Department of Urology, Angers University Hospital, Angers, France, the "I"Comité de Cancérologie de l'Association Française d'Urologie (CCAFU) and the ${ }^{\star \star *}$ Department of Urology, Rennes University Hospital, Rennes, France

Acknowledgements: Pierre I. Karakiewicz is partially supported by the University of Montréal Heath Centre Urology Associates, Fonds de la Recherche en Santé du Québec, the University of Montréal Department of Surgery and the University of Montréal Health Centre (CHUM) Foundation.

This article has been peer reviewed.

Competing interests: None declared.

\section{References}

1. Dhote R, Thiounn N, Debre B, et al. Risk factors for adult renal cell carcinoma. Urol Clin North Am 2004;31:237-47.

2. Pantuck AJ, Zisman A, Belldegrun AS. The changing natural history of renal cell carcinoma. J Urol 2001;166:1611-23.

3. Sanchez-Ortiz RF, Rosser CJ, Madsen LT, et al. Young age is an independent prognostic factor for survival of sporadic renal cell carcinoma. J Urol 2004;171:2160-5.

4. Taccoen X, Valeri A, Descotes JL, et al. A. Renal cell carcinoma in adults 40 years old or less: young age is an independent prognostic factor for cancer-specific survival. Eur Urol 2007;51:980-7.

5. Verhoest $G$, Veillard $D$, Guille $F$, et al. Relationship between age at diagnosis and clinicopathologic features of renal cell carcinoma. [discussion]. Eur Urol 2007;51:1298-304.

6. Minardi AJ Jr, Sittig KM, Zibari GB, et al. Colorectal cancer in the young patient. Am Surg 1998;64:849-53.

7. Parramore JB, Wei JP, Yeh KA. Colorectal cancer in patients under forty: presentation and outcome. Am Surg 1998;64:563-7. 
8. Patard JJ, Leray E, Rioux-Leclercq N, et al. Prognostic value of histologic subtypes in renal cell carcinoma: a multicenter experience. J Clin Oncol 2005;23:2763-71.

9. Patard JJ, Leray E, Rodriguez A, et al. Correlation between symptom graduation, tumor characteristics and survival in renal cell carcinoma. Eur Urol 2003;44:226-32.

10. Lieber MM, Tomera FM, Taylor WF, et al. Renal adenocarcinoma in young adults: survival and variables affecting prognosis. J Urol 1981;125:164-8.

11. Gaidos C, Tartter PI, Bleiweiss IJ, et al. Stage 0 to stage III breast cancer in young women. J Am Coll Surg 2000;190:523-9.

12. Citterio $G$, Bertuzzi $A$, Tresoldi $M$, et al. Prognostic factors for survival in metastatic renal cell carcinoma: retrospective analysis from 109 consecutive patients. Eur Urol 1997; 31:286-91.

13. Rodriguez A, Tazi H, Patard JJ, et al. Renal cell carcinoma in adults less than 40 years of age: A particular cancer? Incidence, outcome and review of the literature. Ann Urol 2003;37:155-9.

14. Gillett MD, Cheville JC, Karnes RJ, et al. Comparison of presentation and outcome for patients 18 to 40 and 60 to 70 years old with solid renal masses. J Urol 2005; 173:1893-6.
15. Pizzocaro $G$, Piva $L$, Colavita $M$, et al. Interferon adjuvant to radical nephrectomy in Robson stages II and III renal cell carcinoma: a multicentric randomized study. J Clin Oncol 2001;19:425-31.

16. Messing EM, Manola J, Wilding G, et al. Phase III study of interferon alfa-NL as adjuvant treatment for resectable renal cell carcinoma: an Eastern Cooperative Oncology Group/Intergroup trial. J Clin Oncol 2003;21:1214-22.

17. Clark JI, Atkins MB, Urba WJ, et al. Adjuvant high-dose bolus interleukin-2 for patients with high-risk renal cell carcinoma: a cytokine working group randomized trial. J Clin Oncol 2003;21:3133-40.

18. Atzpodien J, Schmitt E, Gertenbach U, et al. Adjuvant treatment with interleukin-2- and interferon-alpha2a-based chemoimmunotherapy in renal cell carcinoma post tumour nephrectomy: results of a prospectively randomised trial of the German Cooperative Renal Carcinoma Chemoimmunotherapy Group (DGCIN). Br J Cancer 2005;92:843-6.

Correspondence: Dr. Pierre I. Karakiewicz, Cancer Prognostics and Health Outcomes Unit, University of Montréal Health Centre (CHUM), 1058, rue St. Denis, Montréal, QC H2X 3J4; pierre.karakiewicz@umontreal.ca

\title{
We welcome your comments on the journal and on specific articles.
}

\author{
All letters will be considered \\ for publication in the journal.
}

\section{Send your letters to the Editor-in-Chief at journal@cua.org}

CUAJ JAUC

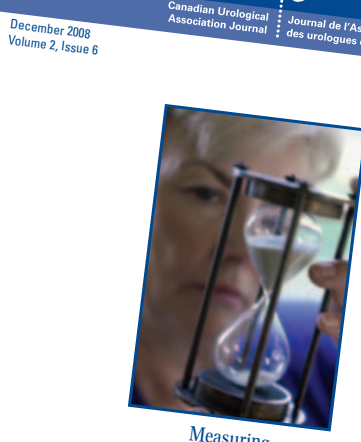

Surgical wait ti

surgical wait times

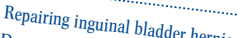

Does oxazepam decreader hernias

Treating lower pole stones: the deba

Décembre 2008
Volume 2 2Numáro 